\title{
Evaluation of a Suspended Personal Radiation Protection System vs. Conventional Apron and Shields in Clinical Interventional Procedures
}

\author{
Clare Savage $^{1 *}$, Thomas M. Seale IV ${ }^{2}$, Cathryn J. Shaw ${ }^{2}$, Bruner P. Angela ${ }^{2}$, \\ Daniel Marichal ${ }^{2}$, Chet R. Rees ${ }^{2}$ \\ ${ }^{1}$ River City Imaging, San Antonio, USA \\ ${ }^{2}$ Department of Radiology, Medical Center, Baylor University, Dallas, USA

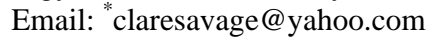

Received May 16, 2013; revised June 16, 2013; accepted June 23, 2013

Copyright (c) 2013 Clare Savage et al. This is an open access article distributed under the Creative Commons Attribution License, which permits unrestricted use, distribution, and reproduction in any medium, provided the original work is properly cited.

\begin{abstract}
Purpose: This clinical study compares conventional lead aprons and ancillary shields to a functionally weightless personal overhead-supported system with expanded coverage. Materials and Methods: Primary operators performed procedures $(\mathrm{N}=126$, fluoroscopy minutes $=1209)$ using one of 2 methods of radiation protection and wearing dosimeters on multiple body locations. Method "LAS" (Lead-Apron+Shields): lead skirt, vest, thyroid shield, with 100\% use of under-table shield, side shield, and mobile suspended lead-acrylic shield. Method "Zgrav": ZeroGravity system (CFI Medical Solutions) with variable use of shielding. The studied early model moving with the operator had a curved lead-acrylic head shield $(0.5 \mathrm{~mm} \mathrm{~Pb})$ and expansive lead apron $(0.5-1.0 \mathrm{~mm} \mathrm{~Pb})$ that covered leg to distal calf and proximal arm to elbow, and a drape that permitted sterile entry and exit. Study was institutional review board approved and HIPPA-compliant. Results: Measured with a sensitive electronic dosimeter, eye exposures were 99\% (P < 0.001) reduced for Zgrav with upgraded face shield vs. LAS, regardless of use or non-use of suspended shield with Zgrav. With optically stimulated luminescence (OSL) dosimeters, operator exposures, standardized to minutes of fluoroscopy and Fluoroscopic Patient Dose Area Product, were reduced by $87 \%-100 \%$ for eye \& head, neck, humerus, and tibia (Zgrav vs. LAS). Overall eye \& head exposure reduction for entire study was $94 \%$. Non-equivalence of torso exposures was not demonstrated. A brief user survey showed ergonomic advantages of Zgrav. Conclusion: Compared to conventional lead aprons with shields, the suspended system provided superior operator protection during interventional fluoroscopy, allowing operators to perform procedures without potentially obstructive shields.
\end{abstract}

Keywords: Radiation Protection; Radiation; Fluoroscopy

\section{Introduction}

The growth in utilization and complexity of fluoroscopic procedures has increased workload for interventionalists, resulting in cumulative radiation doses and orthopedic strains that can be limiting or career ending [1-11]. Despite suffering significantly increased neck and back pain, lost work time, and cervical disc herniations $(\mathrm{P}<0.01)$ [4], exposures remain excessive as demonstrated by one operator receiving the equivalent of 60 skull films during 1 month's practice using available shielding [12]. Data suggest that cataract formation is occurring at exposures less than previously believed with a low or absent threshold dose [5-10]. Lead glasses are often limited to an

"Corresponding author. attenuation factor of 2 - 3 times for the eyes due to angle of coverage relative to the radiation path and backscatter from the unprotected head to the eyes, and are considered heavy, prone to fogging, and incompatible with corrective eyewear [6-14]. Lead glasses do not protect the cervical bone marrow and nervous system where even moderate doses of radiation are associated with elevated incidence of nervous system tumors [1,11,15-17]. Ancillary protection including table shields, hanging leadacrylic shields, and attenuating drapes reduce scatter when positioned properly, but are sometimes awkward and difficult to position due to interference with operator, patient, or image receptor, especially with oblique projections where scatter may increase several-fold $[1,5,6$, 18-21]. These shields produce a discontinuous barrier 
that is not always in the path of scatter and may not be used without a lead apron. As stated by the recent Joint Inter-Society task Force on Occupational Hazards in the Interventional Laboratory, "Interventional physicians and their professional societies, working together with industry, should strive toward the ultimate definition of ALARA as close to a zero radiation exposure work environment as possible, and ultimately eliminate the need for personal protective apparel and prevent its orthopedic and ergonomic consequences" [1].

The suspended personal radiation protection system was designed to enhance radiation protection and improve ergonomics and comfort by eliminating weight on the operator, while maintaining a neutral or positive effect on task accomplishment. The purpose of this study was to assess radiation doses to actual operators during interventional cases using either the suspended system or conventional aprons and ancillary shielding.

\section{Materials and Methods}

Several operators performed procedures in three Interventional Radiology suites (Siemens, Munich, Germany) equipped with 3 ancillary shields (Figure 1), including a mobile suspended lead-acrylic shield, an under-table shield, and a side shield that extends upwards from the under-table skirt (Mavig, Munich, Germany). Primary operators wore multiple dosimeters (described below) while using one of two methods of operator protection: "LAS" (lead apron + shields) and "Zgrav" (suspended radiation protection system).

Method LAS utilized a conventional skirt and vest, thyroid collar, and $100 \%$ use of all 3 types of ancillary shields (Figure 1, Table 1). Most wrap-around skirts and vests were $0.5 \mathrm{~mm} \mathrm{~Pb}$ in front $(1 \mathrm{~mm}$ in frontal area of overlap) and $0.25 \mathrm{~mm} \mathrm{~Pb}$ in back. One used in 6 of 35 LAS cases was $0.25 \mathrm{~mm}$ in the front and back, with 0.5 $\mathrm{mm} \mathrm{Pb}$ in the frontal overlap area. Thyroid shields were $0.5 \mathrm{~mm} \mathrm{~Pb}$ equivalent.

Method Zgrav utilized the suspended personal radiation protection system (ZeroGravity, CFI Medical Solutions, Fenton, MI) with or without ancillary shields according to operator discretion (Figure 2, Table 1). Use of Zgrav vs. LAS was not controlled. The test system was early model commercial stock approved device. This overhead-suspended system had a curved lead-acrylic head shield $(0.5 \mathrm{~mm} \mathrm{~Pb})$, and lead apron extending to the distal calves $(1 \mathrm{~mm} \mathrm{~Pb}$ centrally [63.5 × $69.3 \mathrm{~cm}$ ], 0.5 $\mathrm{mm}$ peripherally) with $0.5 \mathrm{~mm} \mathrm{~Pb}$ arm flaps extending to the elbows. A sterile plastic drape permits quick entry and exit while maintaining sterility. Newer models use thicker $\mathrm{Pb}$ throughout the apron $(1 \mathrm{~mm} \mathrm{~Pb})$.

Operators used patient-exposure reduction techniques including reduction of air gaps, pulsed fluoroscopy, collimation, minimization of fluoroscopy times, minimize-

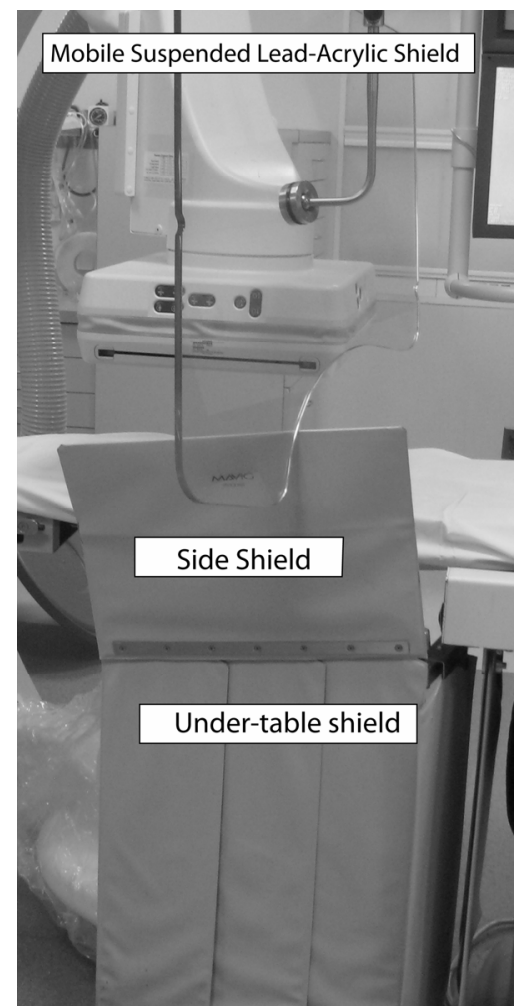

Figure 1. Ancillary shielding consisting of under-table shield, side shield, and mobile suspended lead-acrylic shield were used aggressively in all LAS procedures and sometimes with Zgrav as depicted in Table 1.

Table 1. Ancillary shields used (proportion of procedures).

\begin{tabular}{cccc}
\hline Group & $\begin{array}{c}\text { Hanging } \\
\text { Pb-acrylic }\end{array}$ & Side-table & Under-table \\
\hline LAS & & & \\
All Phases & $100 \%$ & $100 \%$ & $100 \%$ \\
Zgrav & & & \\
Phase I & $<50 \%$ & & $100 \%$ \\
Phase II & & $>50 \%$ & $100 \%$ \\
Eye & $61 \%$ & $>50 \%$ & $100 \%$ \\
Wrist & $0 \%$ & & \\
\hline
\end{tabular}

tion of severely obliqued or lateral receptor angles. Hands are never placed in the direct beam. During most cases, LAS and Zgrav operators stood in the control room or at a distance behind a floor-supported mobile shield during digital subtraction angiography (DSA). The patient was positioned to allow the operator to work at the right side of the table when possible.

Since the left-anterior side of the operator is expected to receive the highest doses, dosimeters were placed accordingly (Figure 3) [12]. The operator wearing the dosimeters was always in the primary operator position.

Optically stimulated luminescence (OSL) dosimeter badges (Landauer Luxel, Glenwood, IL) were placed on 


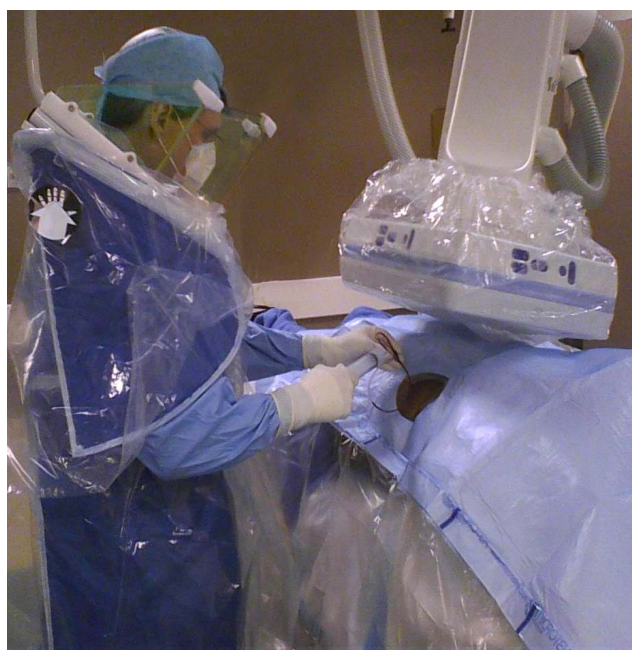

(a)

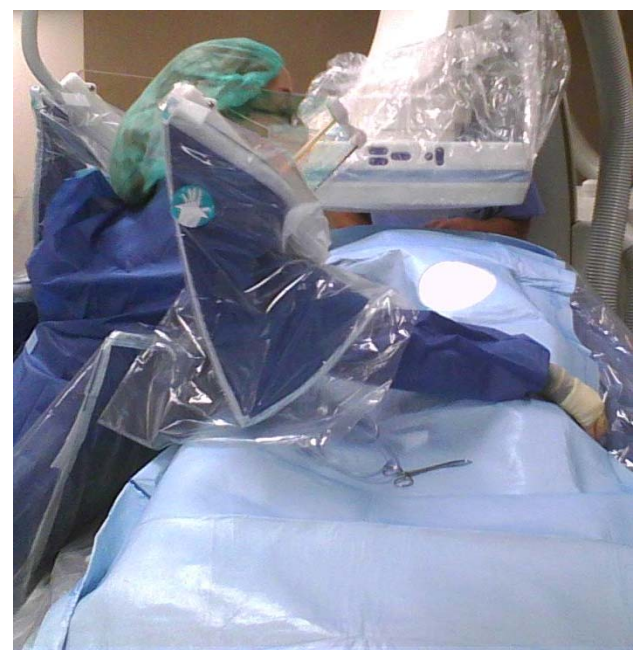

(c)

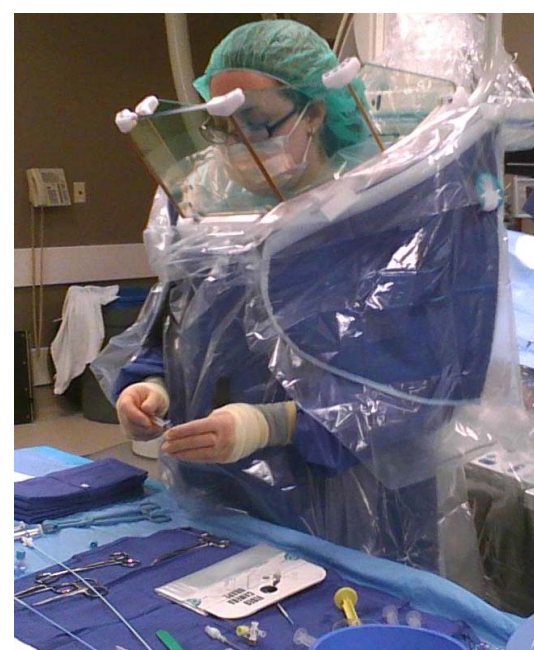

(b)

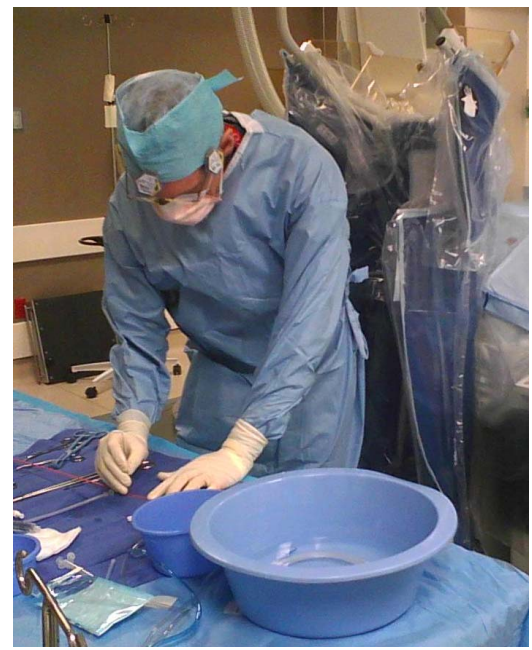

(d)

Figure 2. Zerogravity device in clinical use. Top left (a) Device provides shielding without the challenges of the suspended shield when using left anterior oblique tube angulation during body intervention. Operator looks over the face shield to the monitor. Top right (b) Device mores and turns in the field with the operator. Bottom left (c) Operator is able to lean over large patient. Bottom right (d) operator steps out of device to perform delicate work at back table, and can then re-engage the device for fluoroscopy while maintaining sterility.

operators prior to donning the LAS or Zgrav, in the locations depicted in Figure 3. Three to five control badges were mailed for readings with each set of badges, and their mean was subtracted from the others to correct for background radiation or possible $\mathrm{x}$-ray examination during mailing.

The data in this report is categorized into 2 phases, differing by time and dosimeter type. For more sensitive, per-case determinations of exposure, a recently calibrated electronic direct dosimeter (EDD-30, Unfors, Billdal, Sweden) was acquired for Phase II. It has a small sensor on a wire that could be placed nearly anywhere, providing readings as low as $1 \mathrm{nSv}$. It may be reset for each case so there are no cumulative effects of natural background radiation.
Dosimeters were worn when fluoroscopy was anticipated to exceed 2 minutes. Inclusion into this report occurred when operator wearing badges was the primary operator for the entire procedure without change in position with another operator, and when procedural data and dosimeter data were available. OSL badge data was excluded for 2 sets that were found, after exposure, to have been positioned incorrectly.

Procedure type, fluoroscopy time, and Total Patient Dose-Area-Product $\left(\mathrm{uSv} / \mathrm{Gycm}^{2}\right)$ as measured by the imaging equipment were available for each case. Total Patient Dose-Area-Product (DAP) includes DAP from all sources including fluoroscopy and DSA. Determination of DAP due to fluoroscopy only (Fluoroscopic Patient DAP) was also available in the last 27 of 67 procedures 
in Phase I and all 50 procedures in Phase II as the study was consolidated into one suite that provided this information. In this study, Fluoroscopic Patient DAP provides a better correlate for pertinent scattered radiation due to the operators' habits of exiting the area during DSA (see Discussion). Operator exposure results were standardized to fluoroscopy time, Total Patient DAP, and Fluoroscopic Patient DAP.

A variety of procedures included: arterial diagnostic (lower extremity, renal, visceral, bronchial, subclavian), arterial interventional (hepatic infusion, peripheral, renal, and visceral stent placement, chemo-embolization, embolization of pulmonary and muscular arteriovenous malformations, embolization of visceral, uterine, and translumbar aortic arteries), venous interventional (transjugular and trans-femoral inferior vena cava [IVC] filters, IVC percutaneous transluminal angioplasty, transjugular liver biopsy, transjugular intrahepatic portosystemic shunt, permcath placements), and non-vascular interventional (biliary, nephrostomy, fiducial placements, abscess drain, gastric tube).

Details of the phases follow:

Phase I: Operators performed 67 procedures using Zgrav $(\mathrm{n}=32)$ or LAS $(\mathrm{n}=35)$ wearing multiple OSL dosimeter badges (Figure 3). Study parameters are depicted in Tables 1 and 2. For Zgrav, the mobile suspended and side shields were used in $<50 \%$ of procedures, and the under-table shield was used $100 \%$ of the time. All 3 ancillary shields were used in $100 \%$ of LAS procedures. The Zgrav face shield was upgraded to a more comprehensive, taller version (Figure 2) for the latter 13 Phase I Zgrav patients and throughout Phase II.

Phase II: The data obtained with the Unfors EDD-30 electronic dosimeter constitutes Phase II. The sensor was worn on the eyeglasses frame near the left eye, or on the dorsum of the left wrist (Figure 3, Table 2). The sus- pended lead-acrylic shield was always used for LAS, and used in $61 \%$ and $0 \%$ with Zgrav when the dosimeter was worn on the eye and wrist, respectively (Table 1). Only 1 designated suite was used for phase II, minimizing uncontrolled variables. The upgraded taller face shield (Figure 2) was used throughout phase II. The use of the EDD-30 electronic dosimeter in conjunction with the OSL badges in some procedures created partial overlap between Phases I and II (12 procedures, 82 minutes of fluoroscopy).

Evaluation of ergonomics: Four operators with extensive use of the device, including 3 study participants (CS, $\mathrm{DM}$, and CJS) completed a brief survey regarding the ergonomics of the device.

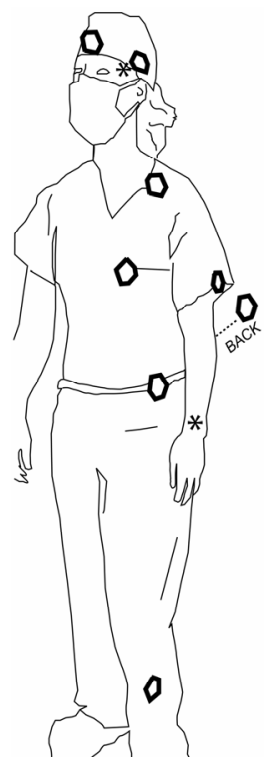

Figure 3. OSL badge dosimeter locations. Asterisks depict the two possible locations for EDD-30 electronic dosimeter in phase II.

Table 2. Other study parameters.

\begin{tabular}{|c|c|c|c|c|c|c|}
\hline & & & & & \multicolumn{2}{|c|}{ Patient DAP $\left(\mathrm{Gy} \mathrm{cm}{ }^{2}\right)$} \\
\hline & Dosimeter Type & Procedures (N) & Operators $(\mathrm{N})$ & Minutes Fluoroscopy & DAP Fluoroscopy & DAP Total \\
\hline Phase I & OSL badges & 67 & & & & \\
\hline Zgrav & & 32 & 3 & 307 & $47.486^{*}$ & 267.801 \\
\hline LAS & & 35 & 3 & 307 & $50.561^{* *}$ & 318.839 \\
\hline Phase II & EDD-30 & & & & & \\
\hline Eye & & 50 & & & & \\
\hline Zgrav & & 28 & 2 & 329 & 103.884 & 281.364 \\
\hline LAS & & 22 & 3 & 122 & 47.734 & 222.364 \\
\hline Wrist & & 21 & & & & \\
\hline Zgrav & & 15 & 2 & 186 & 83.316 & 414.680 \\
\hline LAS & & 7 & 2 & 40 & 22.083 & 131.520 \\
\hline
\end{tabular}

"Data available for last 13 cases, 112 minutes of fluoroscopy. ${ }^{* *}$ Data available for last 14 cases, 132 minutes fluoroscopy. 
Ninety five percent confidence intervals for OSL data were determined based on manufacturer specifications (95\% CI range is $+15 \%$ ) and control readings. In Phase II, one tailed unpaired T-test was used to compare Zgrav and LAS results, and two tailed unpaired T-test was used to compare Zgrav + mobile suspended lead-acrylic shield vs. Zgrav without this shield. Disclosure: CRR has a financial interest in the Zerogravity device.

\section{Results}

Total $\mathrm{N}=126$, fluoroscopy $=1209$ minutes. Eye exposure can be reported for all procedures because it was the only site measured in both phases. It was reduced by 94\% for Zgrav compared to LAS (means $=0.142$ and $2.401 \mathrm{uSv} / \mathrm{minute}$ fluoroscopy, respectively). Figure 4 shows how face shield protects eyes without obstructing line of sight to the monitor.

Phase I: Phase I information and results are depicted in Tables 1, 2, Figures 5(a) and (b). Operator exposures for several body locations were considerably higher for LAS than Zgrav, despite the consistent use of all ancillary shields with LAS (Figures 5(a) and (b)). Operator exposures, standardized to minutes of fluoroscopy (Figure 5(a)) and Fluoroscopic Patient Dose Area Product (Figure 5(b)), were reduced by $87 \%$ - 100\% for eye \& head, neck, humerus, and tibia (Zgrav vs. LAS). Torso and back exposures were low for both modalities (all 95\% confidence intervals include 0 ) so reductions were not calculated. Accurate comparisons for these areas, to determine presence or absence of effect of the $1.0 \mathrm{~mm} \mathrm{~Pb}$ would require data collection on a larger scale. Upper forehead exposures were reduced by both Zgrav faceshield models compared to LAS, however the later model shield was superior (58\% reduction for early model [ $\mathrm{N}=$ 19], $92 \%$ reduction for later model [ $\mathrm{N}=13]$ ).

Results standardized to minutes of fluoroscopy (Figure 5(a), entire Phase I) were similar to results standardized to fluoroscopic patient DAP (Figure 5(b), last 27

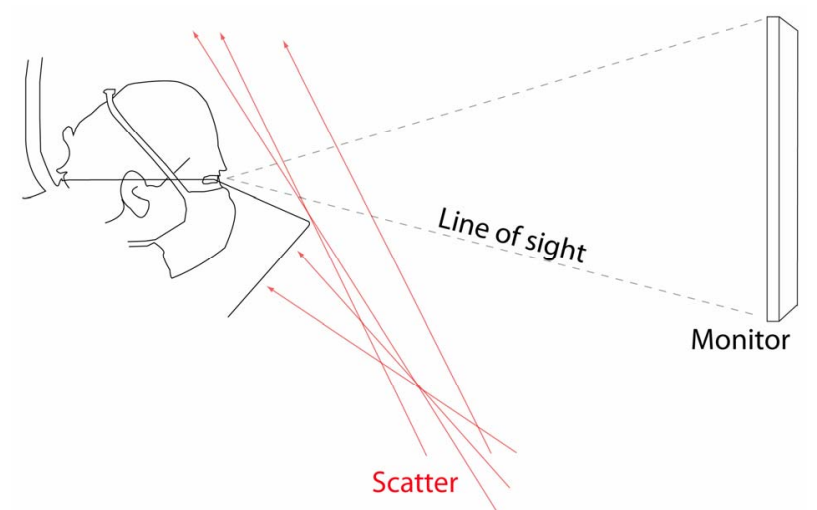

Figure 4. Head shield is in path of scatter but not in line of sight to monitor. patients of Phase I). The similar overall appearances of Figures 5(a) and (b) shows apparent consistency of results for both standardization schemes with regard to exposure reductions (Zgrav vs. LAS) and body part distributions.

Phase II: Information and results for Phase II are seen in Tables 1, 2, and Figure 5(c). Zgrav provided 99\% reductions in Eye exposures $(\mathrm{P}<0.001)$ compared to LAS. The highest exposure in the Zgrav group was lower than the lowest exposure in the LAS group (Figure 5(c)), despite $100 \%$ use of all ancillary shields in the LAS group (Table 1). Exposures for the 28 Zgrav procedures were consistently undetectable or barely detectable, with a mean of $0.007 \mathrm{uSv} / \mathrm{Gycm}^{2}$ (range $=0$ to $0.04 \pm 0.012$ $\mathrm{SD})$, vs 0.767 (0.19 to $4.44 \pm 1.256)$ for LAS $(\mathrm{P}<0.001)$. The strikingly greater variance for LAS compared to Zgrav indicates less consistent shielding for LAS over a broad range of procedure types and operator positions. Finally, Figure 5(c) demonstrates that the use of the mobile suspended lead-acrylic shield did not significantly impact the eye and head exposure when using Zgrav (Mean $+\mathrm{SD}=0.00796 \pm 0.0146 \mathrm{uSv} / \mathrm{Gycm}^{2}$ with shield vs $0.00697 \pm 0.0092$ without shield, $\mathrm{P}=0.14)$. This finding for Zgrav differs from widely reported results for conventional lead aprons where the mobile suspended shield reduced eye exposures [5,6,20,21].

Operator wrist exposures were similar for LAS and Zgrav (Mean $+\mathrm{SD}=2.10 \mathrm{uSv} / \mathrm{Gycm}^{2} \pm 0.75$ vs $2.11 \pm$ 0.76 , $\mathrm{P}>0.05$ ). Since the mobile suspended lead-acrylic shield was used in $100 \%$ of LAS procedures and $0 \%$ of Zgrav procedures in the wrist group (Table 1), this suggests ineffectiveness of the ancillary shields for reducing hand exposures, and that their omission does not increase exposures. These results were consistent with other reports $[20,22,23]$. When standardized to Total DAP (instead of fluoroscopic DAP), wrist exposure for all procedures in Phase II $=0.39 \mathrm{uSv} / \mathrm{Gycm}^{2}$.

The ergonomic survey results for the device were as follows: On a scale of 0 - 5, "relief of back pain normally experienced with a lead apron with $0=$ no relief, and $5=$ complete relief" was a 4 in one person, and not evaluable in 3 persons due to lack of back pain with lead apron. Relative to lead apron, "device comfort" (scale 1 - 5 with 5 = "Much more comfortable" and 1 = "Much less comfortable," and 3 = equal) was 4.75. All responders "always or nearly always use all 3 ancillary shields when using lead apron”. Zgrav was considered less "hassle or obstruction” compared to using all 3 shields and lead apron (4.75 on scale of 1 - 5 with $1=$ Zgrav is much more obstructive, 5 = Zgrav is much less obstructive, $3=$ equal). Procedure time was felt to be "about equal" for Zgrav relative to lead apron for all persons (3 on a scale of 1 - 5). 


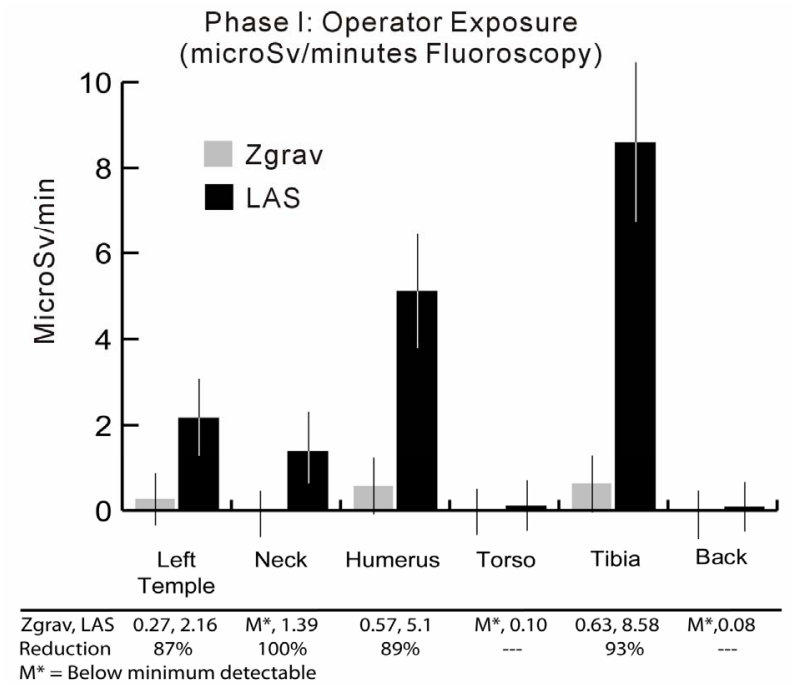

(a)

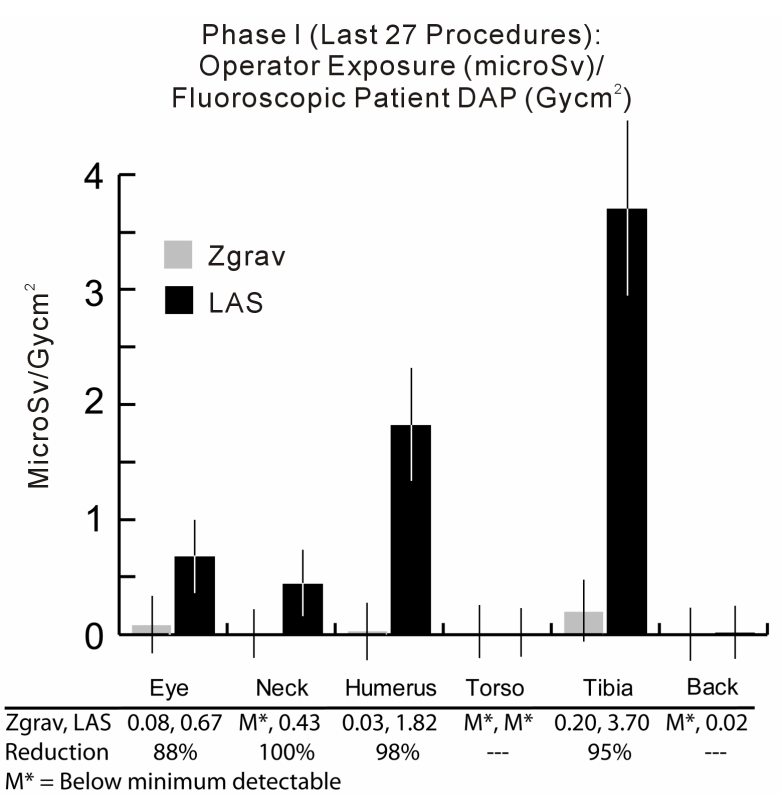

(b)

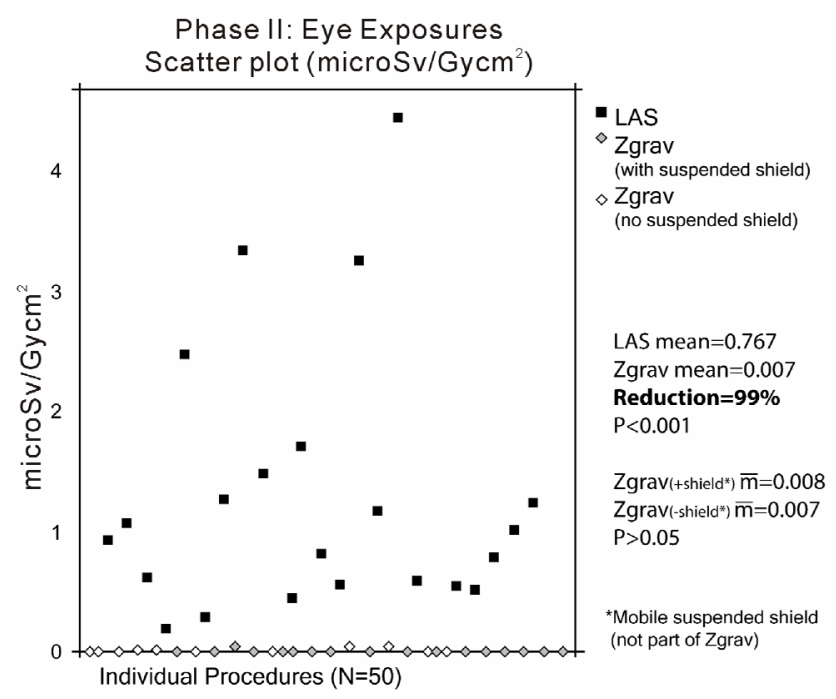

(c)

Figure 5. a. Operator exposures measured with Optically Stimulated Luminescence (OSL) dosimiters and standardized to fluoroscopy minutes in phase I. b. Operator exposures measured with Optically Stimulated Luminescence (OSL) dosimeters and standardized to fluoroscopic DAP in phase I. c. Operator exposures measured with EDD-30 and standardized to fluoroscopic Dose Area Product (DAP) in phase II. Substantial reductions with Zgrav are seen for both standardization techniques and dosimeter types. Depiction of individual cases shows consistency of Zgrav protection relative to LAS and no apparent benefit of mobile suspended lead-acrylic shield when using Zgrav.

\section{Discussion}

The tested device was developed to provide near comprehensive radiation protection sustainable throughout a spectrum of interventional cases. ZeroGravity differs from other weightless lead-apron substitutes by combining additional head shielding, rapid sterile entry/exit, and overhead suspension allowing body motion without floor-base motion $[24,25]$.

The LAS technique, the most broadly studied protec- tion method, is limited by difficulty maintaining ideal shielding position during interventional procedures, and the barrier discontinuity of multiple, separate devices [1,5,6,18-20]. Alternatively, the ZeroGravity provides a single continuous enveloping barrier that shields the top of the head through the lower tibias (broken only by protrusion of the arms through the arm flaps). The device automatically maintains its position between the scatter and the operator as the many dynamic factors change. This effect is demonstrated in Figure 5(c), where ex- 
treme variability in eye exposures in the LAS group occurred despite aggressive use of the mobile suspended lead-acrylic shield in all cases, while eye exposures in the Zgrav group were consistently substantially lower. These differences are unlikely due to material properties since both the mobile suspended shield and the Zgrav face shield use similar Pb-acrylic (0.5 mm Pb).

Combinations of lead apron, attenuating drapes, accessory shields and glasses have been studied using phantoms to extrapolate radiation protection theoretically provided to the practicing interventionalist $[13,14,20$ 22,26,27]. Although phantom studies provide preliminary information, application to clinical practice is problematic due to many dynamic factors including large patients, interference with operator's hands or arms from accessory shields, and inability to use shielding in certain image receptor obliquities or operator stances. Results with phantoms showing significantly reduced eye exposures using shields are not fully corroborated by clinical studies using the same shielding, with one study documenting high clinical eye doses and recommending protective eyewear in addition to the suspended shield [20-23,27-29]. A feasibility study of an earlier prototype of the suspended personal radiation protection system using phantoms showed 16 - 78 fold decreases in exposure to various body areas compared to a lead apron due to thicker lead and greater surface area covered [30].

Without eye protection, a standard workload could easily exceed the annual limit of $150 \mathrm{mSv}$, and could be further compounded for interventionalists who become patients and receive additional medical exposures [31]. The concurrent use of the mobile suspended lead-acrylic shield produced no detectable differences in eye and head exposures in the Zgrav group (Figure 5(c)), supporting our current practice of abandoning the cumbersome suspended shield, and sometimes forgoing the side shield when obstructive to work. When wearing conventional lead aprons, aggressive use of available ancillary shields is recommend.

To facilitate comparison across the literature, operator exposure was standardized to Total Patient DAP, which is a widely accepted reporting method that reflects the protective power of the shielding, and minimizes effects of uncontrolled variables [22,29,32,33]. Similar to our LAS group, other reports showed high case-to-case variance of DAP-standardized operator exposures due to the dynamic factors of clinical practice [29]. Figure 6 shows our LAS group used shielding very effectively compared to other DAP-standardized reports, attributable to rigorous optimization of shielding when not using the ZeroGravity system.

Further standardization to Fluoroscopic Patient DAP and strict adherence to data from only the primary operator position provide a more pure comparison of Zgrav
Operator head exposures/Total Patient Dose-Area-Product

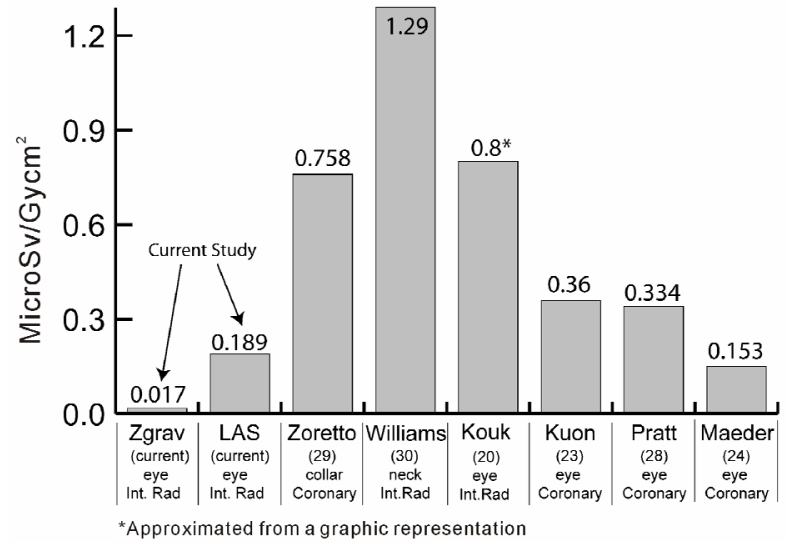

Figure 6. Operator head exposures per Total Patient DAP in clinical studies with mobile suspended lead-acrylic shields. LAS group of current study shows exposures in the low range of similar studies, indicating aggressive use of ancillary shields with lead aprons by operators in current study. Zgrav group of current study shows substantially lower exposures than all studies using conventional means. Dosimeter location is indicated under each study, and procedure type is at bottom.

to LAS. Due to the operators' practice of exiting the area during DSA, Patient DAP during DSA is irrelevant to operator exposure, yet accounted for 2.8 times the contribution of Fluoroscopic Patient DAP towards Total Patient DAP (Table 2). Pure primary operator exposures are difficult to obtain in teaching institutions and unconfirmed in many reports, but are important since secondary operators are farther from the source and partially shielded by the primary operator, showing relative reductions of $50 \%$ [29].

DAP Standardized wrist exposures were similar to other reports. Absence of significant difference between LAS and Zgrav was unsurprising since neither Zgrav nor the mobile suspended lead-acrylic shield protect the hands or forearms [20,22,23]. In keeping with ALARA, we perform $100 \%$ of procedures without ever placing the fingers in the direct beam.

Detailed analysis of the device's ability to improve operator comfort and relieve strain is beyond the study's scope. However, survey results suggested that, compared to lead aprons and all 3 ancillary shields, the suspended personal radiation protection system was more comfortable, relieved back pain in the one afflicted responder, was less obstructive to work effort than the other shields and did not affect procedure time.

This study has potential limitations. The wide case variety, representative of an actual interventional practice, makes cohort matching difficult. The study does not include all consecutive cases for any operator or lab due largely to the occurrences of change in operator status 
(primary vs. secondary) at a teaching institution resulting in exclusions. This study was not powered to detect potential differences in under-lead exposures from different $\mathrm{Pb}$ equivalencies for Zgrav vs. LAS. A larger multi-center study by Marx, et al., showed that, beside case volume, double lead thickness was the single largest determinant of under-lead exposure and more important than ancillary shielding, with $1 \mathrm{~mm}$ lead equivalent aprons resulting in a $2 / 3$ reduction in measured under-lead exposures [34]. Another study showed reductions of $78 \%$ to $83 \%$ using thicker lead to protect the torso [22]. Finally, study results may differ for operators who stay in the area during DSA sequences, receiving higher exposures with different energy spectra.

\section{Conclusion}

In conclusion, the test device significantly reduced exposures for many body areas compared to rigorous conventional shielding techniques in the clinical setting. It is a single, weightless apparatus protecting a great proportion of the body in various operator, patient and tube configurations. Future studies could include in-depth evaluation of its ergonomic benefits, and analysis of cost effectiveness in light of its possible substitution for other ceiling-suspended apparatus.

\section{REFERENCES}

[1] L. W. Klein, D. L. Miller, S. Balter, et al., "Occupational Health Hazards in the Interventional Laboratory: Time for a Safer Environment," Journal of Vascular and Interventional Radiology, Vol. 20, No. 2, 2009, pp. 147-153. doi:10.1016/j.jvir.2008.10.015

[2] N. Hidajet, P. Wüst, M. Kreuschner, et al., "Radiation Risks for the Radiologist Performing Transjugular Intrahepatic Portosystemic Shunt (TIPS),” British Journal of Radiology, Vol. 79, No. 942, 2006, pp. 483-486. doi:10.1259/bjr/67632946

[3] Z. J. Haskal, "Interventional Radiology Carries Occupational Risks for Cataracts,” RSNA News, Vol. 14, 2004, pp. 5-6.

[4] A. M. Ross, J. Segal, D. Borenstein, et al., "Prevalence of Spinal Disc Disease among Interventional Cardiologists," American Journal of Cardiology, Vol. 79, No. 1, 1997, pp. 68-70. doi:10.1016/S0002-9149(96)00678-9

[5] B. A. Schueler, "Operator Shielding: How and Why," Techniques in Vascular and Interventional Radiology, Vol. 13, No. 3, 2010, pp. 167-171. doi:10.1053/j.tvir.2010.03.005

[6] D. L. Miller, E. Vano, G. Bartal, et al., "Occupational Radiation Protection in Interventional Radiology: A Joint Guideline of the Cardiovascular and Interventional Radiology Society of Europe and the Society of Interventional Radiology,” Journal of Vascular and Interventional Radiology, Vol. 21, No. 5, 2010, pp. 607-615. doi:10.1016/j.jvir.2010.01.007
[7] B. V. Worgul, Y. I. Kundiyev, N. M. Sergiyenko, et al., "Cataracts among Chernobyl Clean-Up Workers; Implications Regarding Permissible Eye Exposures,” Vol. 167, No. 2, 2007, pp. 233-243. doi:10.1667/RR0298.1

[8] E. Nakashima, K. Neriishi and A. Minamoto, "A Reanalysis of Atomic-Bomb Cataract Data, 2000-2002: A Threshold Analysis," Health Physics, Vol. 90, 2006, pp. 154-160. doi:10.1097/01.HP.0000175442.03596.63

[9] K. Neriishi, E. Nakashima, A. Minamoto, et al., "Postoperative Cataract Cases among Atomic Bomb Survivors: Radiation Dose Response and Threshold," Radiation Research, Vol. 168, No. 4, 2007, pp. 404-408. doi:10.1667/RR0928.1

[10] National Council on Radiation Protection and Measurements, "Radiation Dose Management for Fluoroscopically-Guided Interventional Medical Procedures,” Report No. 168, 21 July 2010, p. 26.

[11] M. M. Finkelstein, "Is Brain Cancer an Occupational Disease in Cardiologists?” Canadian Journal of Cardiology, Vol. 14, 1998, pp. 1385-1388.

[12] D. B. Wilson, R. A. Becker, R. G. Molnar, et al., "The Cranial Radiation Exposure of Vascular Interventionalists,” Journal of Vascular Surgery, Vol. 51, No. 6, 2010, pp. 67S-68S. doi:10.1016/j.jvs.2010.02.179

[13] A. J. Cousin, R. B. Lawdahl, D. P. Chakraborty, et al., "The Case for Radioprotective Eyewear/Facewear. Practical Implications and Suggestions," Investigative Radiology, Vol. 22, 1987, pp. 688-692. doi:10.1097/00004424-198708000-00012

[14] W. Moore, G. Ferguson and C. Rohrmann, "Physical Factors Determining the Utility of Radiation Safety Glasses," Medical Physics, Vol. 7, No. 1, 1980, pp. 8-12. doi:10.1118/1.594661

[15] D. L. Preston, E. Ron, S. Yonehara, et al., "Tumors of the Nervous System and Pituitary Gland Associated with Atomic Bomb Radiation Exposure," Journal of the $\mathrm{Na}$ tional Cancer Institute, Vol. 94, No. 20, 2002, pp. 15551563. doi:10.1093/jnci/94.20.1555

[16] L. Hardell, K. Hansson Mild, A. Pahlson, et al., "Ionizing Radiation, Cellular Telephones and the Risk for Brain Tumours,” European Journal of Cancer Prevention, Vol. 10, No. 6, 2001, pp. 523-529. doi:10.1097/00008469-200112000-00007

[17] S. Yonehara, A. V. Brenner, M. Kishikawa, et al., "Clinical and Epidemiologic Characteristics of First Primary Tumors of the Central Nervous System and Related Organs among Atomic Bomb Survivors in Hiroshima and Nagasaki, 1958-1995,” Cancer, Vol. 101, No. 7, 2004, pp. 1644-1654. doi:10.1002/cncr.20543

[18] V. Tsapaki, S. Kottou, E. Vano, et al., "Correlation of Patient and Staff Doses in Interventional Cardiology," Radiation Protection Dosimetry, Vol. 117, No. 1-3, 2005, pp. 26-29. doi:10.1093/rpd/nci705

[19] E. Vano, L. Gonzalez, F. Beneytez, et al., "Lens Injuries Induced by Occupational Exposure in Non-Optimized Interventional Radiology Laboratories,” British Journal of Radiology, Vol. 71, 1998, pp. 728-733. 
[20] C. Koukorava, E. Carinou and G. Simantirakis, "Doses to Operators during Interventional Radiology Procedures: Focus on Eye Lens and Extremity Dosimetry,” Radiation Protection Dosimetry, Vol. 10, 2010, pp. 1093-1097.

[21] R. H. Thornton, L. T. Dauer, J. P. Altamirano, et al., "Comparing Strategies for Operator Eye Protection in the Interventional Radiology Suite,” Journal of Vascular and Interventional Radiology, Vol. 21, No. 11, 2010, pp. 1703-1707. doi:10.1016/j.jvir.2010.07.019

[22] E. Kuon, M. Günther, O. Gefeller, et al., "Standardization of Occupational Dose to Patient DAP Enables Reliable Assessment of Radiation-Protection Devices in Invasive Cardiology,” Fortschr Röntgenstr, Vol. 175, No. 11, 2003, pp. 1545-1550. doi:10.1055/s-2003-43412

[23] M. Maeder, H. P. Brunner-La Rocca, T. Wolbwer, et al., "Impact of a Lead Glass Screen on Scatter Radiation to Eyes and Hands in Interventional Cardiologists," Catheterization and Cardiovascular Interventions, Vol. 67, No. 1, 2006, pp. 18-23. doi:10.1002/ccd.20457

[24] O. Dragusin, R. Weerasooriya, P. Jais, et al., "Evaluation of a Radiation Protection Cabin for Invasive Electrophysiological Procedures,” European Heart Journal, Vol. 28, No. 2, 2007, pp. 183-189. doi:10.1093/eurheartj/ehl420

[25] D. M. Pelz, "Low Back Pain, Lead Aprons, and the Angiographer,” American Journal of Neuroradiology, Vol. 21, No. 7, 2000, pp. 1364.

[26] B. A. Schueler, T. J. Vrieze, H. Bjarnason, et al., “An Investigation of Operator Exposure in Interventional Radiology,” Radiographics, Vol. 26, 2006, pp. 1533-1541. doi:10.1148/rg.265055127

[27] T. A. Pratt and A. J. Shaw, "Factors Affecting the Radiation Dose to the Lens of the Eye During Cardiac Catheterization Procedures," British Journal of Radiology, Vol. 66, No. 784, 1993, pp. 346-350.

\section{doi:10.1259/0007-1285-66-784-346}

[28] M. Zorzetto, G. Bernardi, G. Morocutti, et al., "Radiation Exposure to Patients and Operators during Diagnostic Catheterization and Coronary Angioplasty,” Catheterization and Cardiovascular Diagnosis, Vol. 40, No. 4, 1997, pp. 348-351.

doi:10.1002/(SICI)1097-0304(199704)40:4<348::AID-C CD4>3.0.CO;2-9

[29] J. R. Williams, "The Interdependence of Staff and Patient Doses in Interventional Radiology," British Journal of Radiology, Vol. 70, 1997, pp. 498-503.

[30] D. A. Marichal, T. Anwar, D. Kirsch, et al., "Comparison of a Suspended Radiation Protection System versus Standard Lead Apron for Radiation Exposure of a Simulated Interventionalist,” Journal of Vascular and Interventional Radiology, Vol. 22, No. 4, 2011, pp. 437-442. doi:10.1016/j.jvir.2010.12.016

[31] E. Vano, L. Gonzales, J. M. Fernández, et al., "Eye Lens Exposures to Radiation in Interventional Suites: Caution Is Warranted,” Radiology, Vol. 248, 2008, pp. 945-953. doi:10.1148/radiol.2482071800

[32] A. Servomaa and J. Karppinen, "The Dose-Area Product and Assessment of the Occupational Dose in Interventional Radiology,” Radiation Protection Dosimetry, Vol. 96, No.1-3, 2001, pp. 235-236. doi:10.1093/oxfordjournals.rpd.a006590

[33] N. W. Marshall and K. Faulkner, "The Dependence of the Scattered Radiation Dose to Personnel on Technique Factors in Diagnostic Radiology,” British Journal of Radiology, Vol. 23, 1996, pp. 1271-1276.

[34] M. V. Marx, L. Niklason and E. Mauger, “Occupational Radiation Exposure to Interventional Radiologists: A Prospective Study,” Journal of Vascular and Interventional Radiology, Vol. 3, No. 4, 1992, pp. 597-606. doi:10.1016/S1051-0443(92)72903-0 